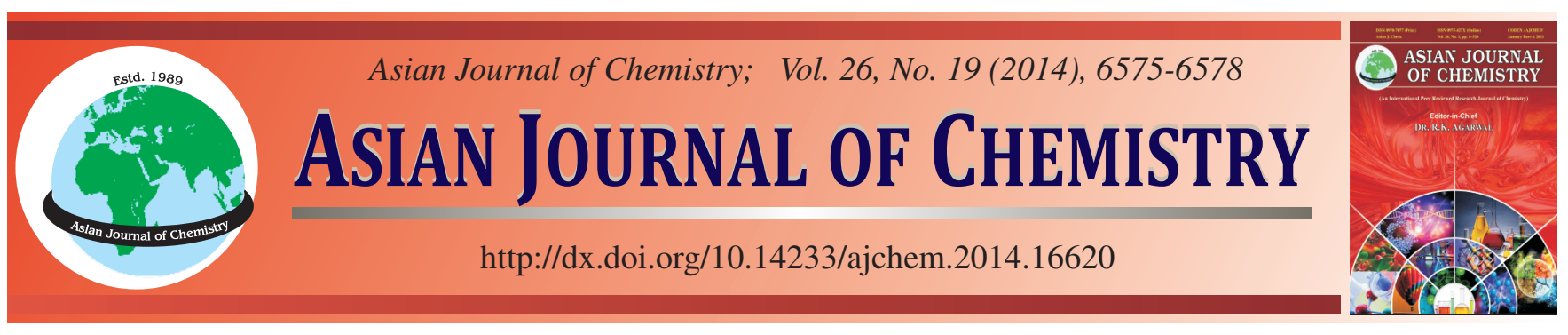

\title{
Swelling and Dissolution Mechanism of Poly(paraphenylene benzobisoxazole) Fibers in Poly Phosphoric Acid
}

\author{
Tian Yu and XiwEN Wang*
}

State Key Lab of Pulp and Paper Engineering, South China University of Technology, Wushan Road No. 381, Guangzhou, P.R. China

*Corresponding author: E-mail: wangxw@ scut.edu.cn

\begin{abstract}
Swelling and dissolution properties of (poly-paraphenylene benzobisoxazole) (PBO) fibers in (poly phosphoric acid) solutions were studied in this paper. The swelling and dissolution process was observed by light microscope and SEM. Depending on concentration of polyphosphoric acid solutions and swelling time, uniform or uneven swelling, disintegration swelling and dissolution were observed. In 5 and $10 \%$ polyphosphoric acid containing solutions, the swelling of fibers were uniform type. In 15 and $20 \%$ containing poly phosphoric acid solutions, the swelling of fibers were disintegration type. The uniform swelling would change to uneven swelling when the fiber immersed in solution for a few more minutes. The poly(paraphenylene benzobisoxazole) fiber could dissolve totally in 5-20 \% polyphosphoric acid/alcohol solution with different time. The swelling process could divide into constant speed dissolution and in constant speed dissolution. Polyphosphoric acid/alcohol solutions resulted in either dissolution or limited swelling depending on polyphosphoric acid concentration and swelling time.
\end{abstract}

Keywords: Poly(paraphenylene benzobisoxazole) fiber, Swelling, Polyphosphoric acid.

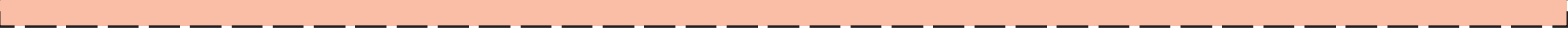

\section{INTRODUCTION}

Poly(paraphenylene benzobisoxazole) (PBO) fibers, aromatic heterocyclic rigid-rod polymeric fibers, have a wide application in military and business fields because they exhibit excellent mechanical property, high flame resistance, high thermal stability and good resistance to creep, chemicals and abrasion $^{1-5}$. Especially, poly(paraphenylene benzobisoxazole) fibers alone or blended with other fibers are used to fabricate composites with adhesive in the form of fabric. Fabric composites are attractive because they are lighter, stiffer, stronger anti-impact force and more flexible than conventional polymers and metals. The additional advantage is that their properties and forms can meet the needs of specific applications such as bullet proof composite material and advanced bearing liner materials.

A lot of work has been done about the surface modification of poly(paraphenylene benzobisoxazole) fiber due to its chemical inert characters ${ }^{6-9}$. However, the stability of poly(paraphenylene benzobisoxazole) fiber under hydrolytic conditions has never been reported. It is difficult to study the hydrolytic stability of poly(paraphenylene benzobisoxazole) fibers because this fiber is completely insoluble in water and have very low solubility in common organic solvents. In this article, swelling and dissolution properties of poly(paraphenylene benzobisoxazole) in polyphosphoric acid (PPA)-alcohol system was studied. The morphological changes of poly(paraphenylene benzobisoxazole) fibers during swelling and dissolution were found. The swelling condition of fibers can accelerate the aging of fiber.

\section{EXPERIMENTAL}

Poly(paraphenylene benzobisoxazole) fiber: Zylon HM type, purchased from Toyobo Ltd. Co., Japan. The fibers were cut into $5 \mathrm{~mm}$ length and were cleaned with ethanol and distilled water respectively and then dried in a vacuum oven at $100{ }^{\circ} \mathrm{C}$ for $12 \mathrm{~h}$. Analytical grade polyphosphoric acid and alcohol were used.

Measurement of fiber diameter: The diameter of swollen fiber was measured by means of Reichert projection microscope with a magnification of 400 times. The swelling rate of poly(paraphenylene benzobisoxazole) fiber was obtained by measuring the fiber diameter after its swelling for varying times: 5, 10, 15, 20, 25, 30, 45, $60 \mathrm{~min}$. Ten fibers were counted and mean value was taken for measurement. '-1' refers to dramatic swelling showing fiber diameter lower than $5 \mu \mathrm{m}$, or refers to the disintegration of the fiber structure and the sign ' -2 ' refers to the dissolution of the fiber for which the fiber diameter could not be measured. 
Morphological change observation: The morphological changes of poly(paraphenylene benzobisoxazole) fibers were observed by light microscope (OLYMPUS, DP70) and scanning electron microscope (SEM, LEO 1530VP, Germany).

\section{RESULTS AND DISCUSSION}

Morphological observation: Figs. 1 and 2 show the morphological changes of poly(paraphenylene benzobisoxazole) fiber in polyphosphoric acid/alcohol solutions. The results indicated that the effect of polyphosphoric acid concentration on poly(paraphenylene benzobisoxazole) fiber can be divided into four type: (1) uniform swelling of fiber at a low polyphosphoric acid content (5 and $10 \%$ ) in alcohol (Fig. 1: A); (Fig. 1: A); (2) uneven swelling along the fiber axis (Fig. 1: B, 2: B) which is followed by uniform swelling; (3) disintegration swelling (Fig. 1: C, 2: C) at a high polyphosphoric acid content (15 and $20 \%$ ) or swelling time extended; (4) dissolution at 5-20\% polyphosphoric acid content for different time (Fig. 1: $\mathrm{D}$ and $\mathrm{F})$.

In Fig. 1, photos A and B show time-dependent swelling in that the uniform swelling of the fiber along the fiber axis proceeds to uneven swelling with time increased. This type of swelling is observed in both 5 and $10 \%$ polyphosphoric acid/ alcohol solutions. Photo $\mathrm{C}$ is the disintegration swelling of a poly(paraphenylene benzobisoxazole) fiber, which was observed in 5-20\% polyphosphoric acid/alcohol solutions. Photos D and $\mathrm{F}$ are the dissolution of poly(paraphenylene benzobisoxazole) fiber, which were found to occur in 5-20\% polyphosphoric acid/alcohol solutions at different time.

In Fig. 2, photo $\mathrm{A}$ is the original poly(paraphenylene benzobisoxazole) fiber, which surface is very smooth. Photo $\mathrm{B}$ is uneven swelling type. The crack and fragment of fiber were observed. Photo $\mathrm{C}$ is the disintegration swelling of poly(paraphenylene benzobisoxazole) fiber. The skin of the fiber almost dissolved in this type and split was founded on the fiber surface.

Effect of polyphosphoric acid concentration on swelling rate of poly(paraphenylene benzobisoxazole) fiber: The effect of polyphosphoric acid concentration on fiber diameter of poly(paraphenylene benzobisoxazole) fiber was shown in Fig. 3. Fig. 3 illustrates the effect of polyphosphoric acid/ alcohol solution having 0- $20 \%$ polyphosphoric acid on the fiber diameter of poly(paraphenylene benzobisoxazole) fibers within 2 min of swelling. When the polyphosphoric acid

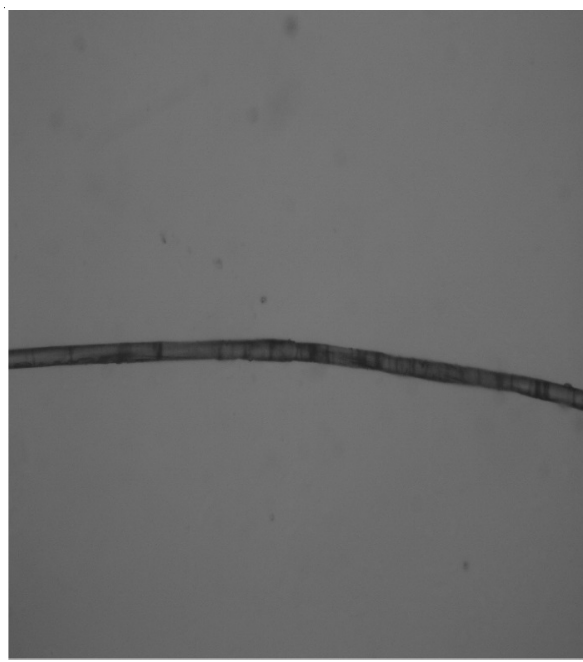

(A)

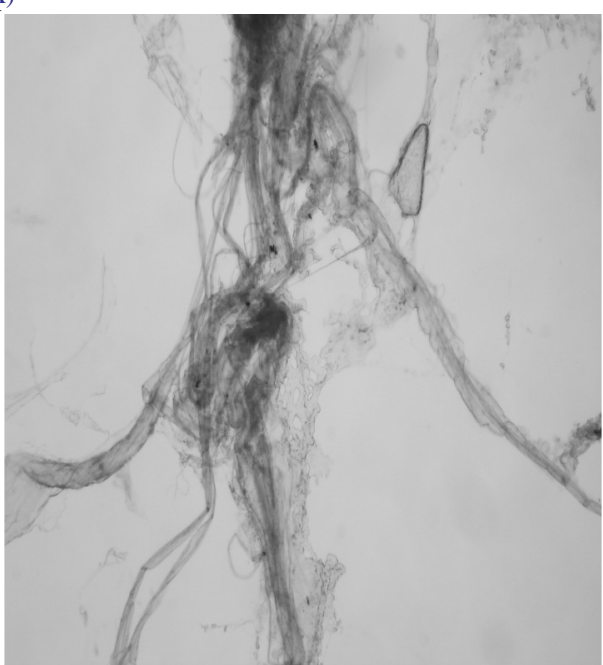

(D)

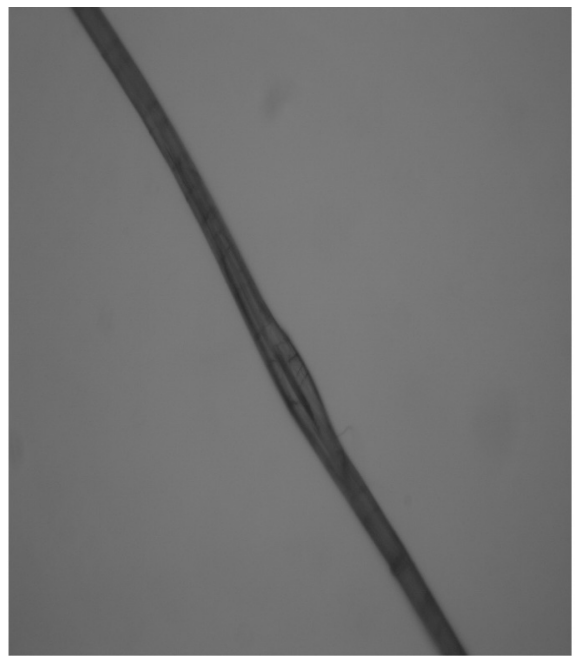

(B)

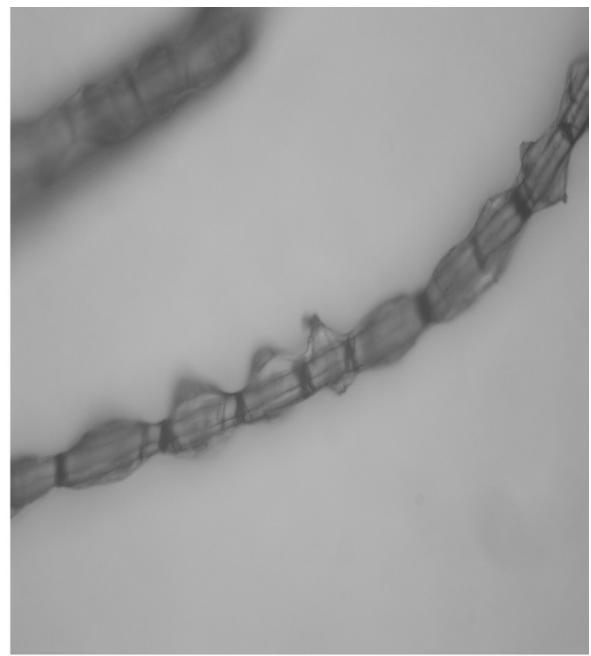

(C)

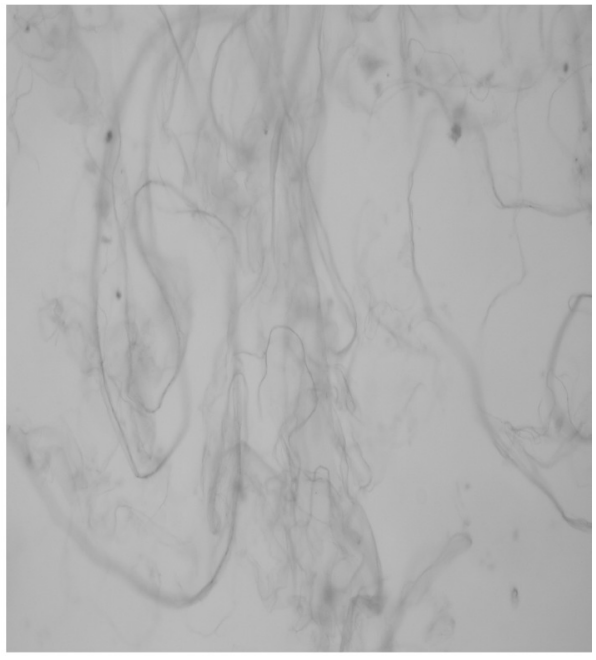

(E)

Fig. 1. Morphology of poly-paraphenylene benzobisoxazole (PBO) fiber in poly phosphoric acid/alcohol solution 


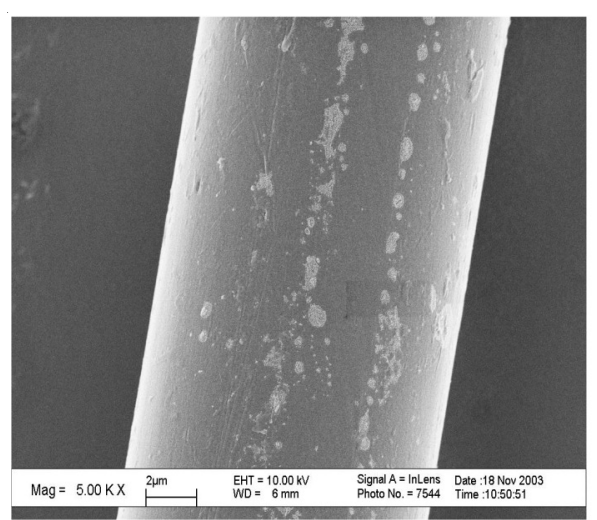

(A)

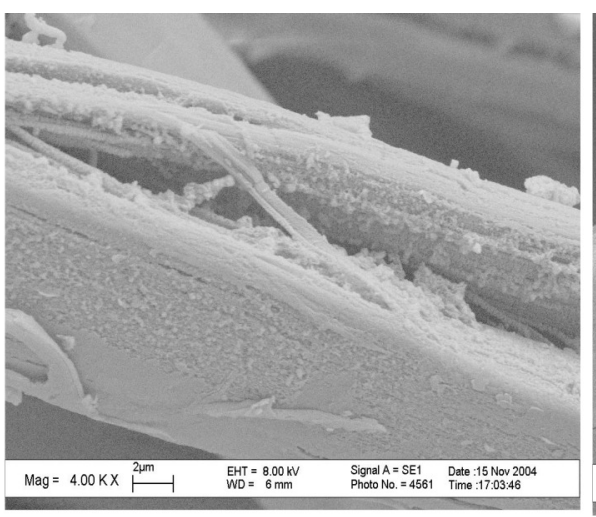

(B)

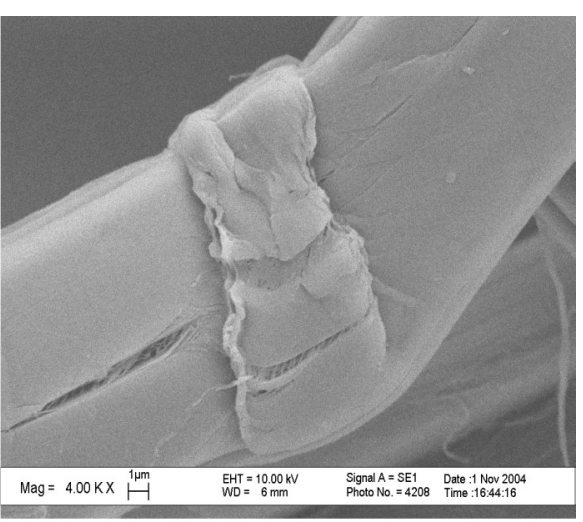

(C)

Fig. 2. SEM observation of swelling poly-paraphenylene benzobisoxazole fiber

concentration increased to $25 \%$, the viscosity of the solution was high. It was difficult to mix polyphosphoric acid and alcohol evenly. So the concentration of polyphosphoric acid/ alcohol solution in this paper was selected 5-20\%. Fiber diameter of original poly(paraphenylene benzobisoxazole) fiber was $12 \mu \mathrm{m}$. Fiber diameter increased at low concentration of polyphosphoric acid (5 and $10 \%$ ). Poly(paraphenylene benzobisoxazole) fiber was swelled firstly at the low polyphosphoric acid concentration. The swelling type is uniform swelling. When the concentration increased to $15-20 \%$, fiber diameter decreased compared to the original fiber. The swelling type is disintegration swelling type at these concentrations. poly(paraphenylene benzobisoxazole) fiber began to dissolve and disintegrate alcohol solution. So the fiber diameter would decrease in the solution.

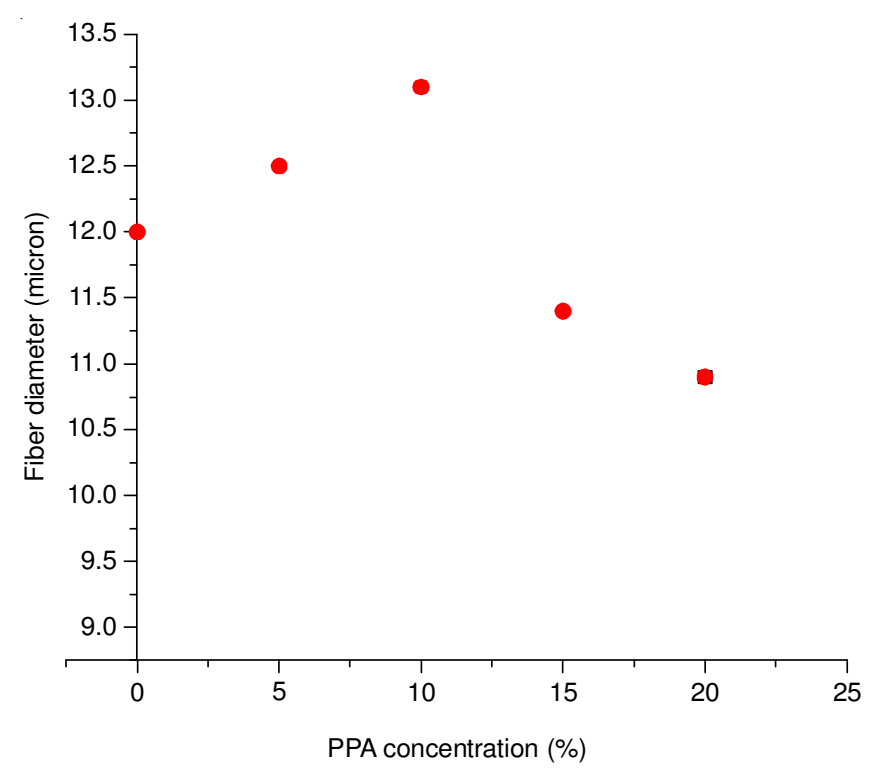

Fig. 3. Effect of polyphosphoric acid concentration of fiber diameter

Effect of swelling time on swelling rate of poly(paraphenylene benzobisoxazole) fiber: Effect of swelling time on swelling rate of poly(paraphenylene benzobisoxazole) fiber was shown in Fig. 4. Fig. 4 showed that poly(paraphenylene benzobisoxazole) fiber would dissolve totally within $1 \mathrm{~h}$ at 5-20\% polyphosphoric acid/alcohol solution with different swelling rate and swelling process. The swelling rate of poly(paraphenylene benzobisoxazole) fiber was accelerated with polyphosphoric acid concentration increased. In $20 \%$ polyphosphoric acid/alcohol solution, poly(paraphenylene benzobisoxazole) fiber dissolved totally in $25 \mathrm{~min}$. However, in $5 \%$ polyphosphoric acid/alcohol solution, time increased to $55 \mathrm{~min}$. The swelling process could be divided into two sorts: (1) constant speed dissolution at the 5 and $10 \%$ polyphosphoric acid concentration; (2) in constant speed dissolution at the 15 and $20 \%$ polyphosphoric acid concentration.

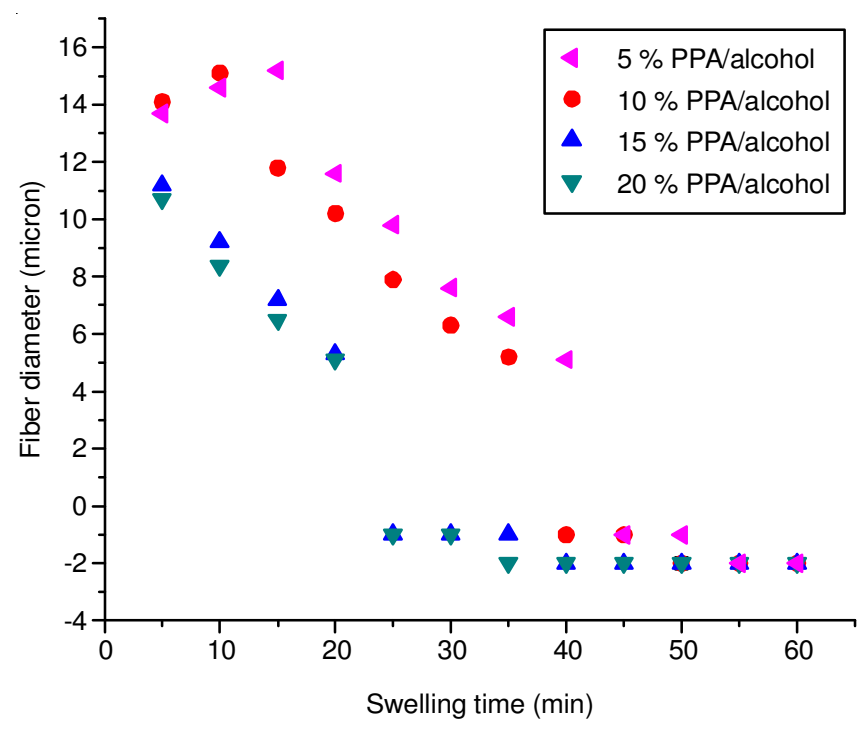

Fig. 4. Effect of swelling time on swelling rate of poly(paraphenylene benzobisoxazole) fiber

\section{Conclusions}

Swelling and dissolution mechanism of poly(paraphenylene benzobisoxazole) fibers in polyphosphoric acid/alcohol solutions is found to relate to swelling time, concentrations. An increase concentration of polyphosphoric acid/alcohol solutions containing:

(1) uniform swelling of fiber at a low polyphosphoric acid content (5 and $10 \%$ ) in alcohol; (2) uneven swelling along the fiber axis which is followed by uniform swelling; (3) disintegration swelling at a high polyphosphoric acid content $25 \%$ or swelling time extended; (4) dissolution at 5-20\% polyphosphoric acid content for different time. The swelling rate of poly(paraphenylene benzobisoxazole) fiber was 
accelerated with polyphosphoric acid concentration increased. The swelling process could be divided into two sorts: (1) constant speed dissolution at the 5 and $10 \% 30$ polyphosphoric acid concentration; (2) inconstant speed dissolution at the 15 and $20 \%$ polyphosphoric acid concentration.

\section{REFERENCES}

1. R.J. Davies, M.A. Montes-Moran, C. Riekel and R.J. Young, J. Mater Sci., 36, 3079 (2001).

2. J.M. Park, D.S. Kim and S.R. Kim, J. Colloid Interf. Sci., 264, 431 (2003).
3. C.H. Zhang, Y.D. Huang and Y.D. Zhao, Mater. Chem. Phys., 268, 127 (2003).

5. G.M. Wu and C.H. Chang, Vacuum, 81, 1159 (2007).

6. X.L. Pan, R.Y. Zhang, S.J. Peng and Y. Qiu, Fibers and Polymers., 11, 372 (2010)

7. T. Zhang, D.Y. Hu, J.H. Jin, S. Yang, G. Li and J. Jiang, Appl. Surf. Sci., 256, 2073 (2010)

8. C.S. Zhang, P. Chen, B.L. Sun, C. Lu, X. Zhang and D. Liu, J. Appl. Polym. Sci., 113, 71 (2009).

9. G.M. Wu, Mater. Chem. Phys., 85, 81 (2004). 